\title{
First detection and genetic characterization of canine Kobuvirus in domestic dogs in Thailand
}

\author{
Kamonpan Charoenkul ${ }^{1,2}$, Taveesak Janetanakit ${ }^{1,2}$, Supassama Chaiyawong ${ }^{1,2}$, Napawan Bunpapong ${ }^{1,3}$, \\ Supanat Boonyapisitsopa ${ }^{1,2}$, Ratanaporn Tangwangvivat ${ }^{1,2}$ and Alongkorn Amonsin ${ }^{1,2^{*}}$ (1)
}

\begin{abstract}
Background: Canine Kobuvirus (CaKoV) has been detected both in healthy and diarrheic dogs and in asymptomatic wild carnivores. In this study, we conducted a survey of CaKoV at small animal hospitals in Bangkok and vicinity of Thailand during September 2016 to September 2018.

Results: Three hundred and seven rectal swab samples were collected from healthy dogs $(n=55)$ and dogs with gastroenteritis symptoms $(n=252)$. Of 307 swab samples tested by using one-step RT-PCR specific to 3D gene, we found CaKoV positivity at $17.59 \%$ (54/307). CaKoVs could be detected in both sick (19.44\%) and healthy (9.09\%) animals. In relation to age group, CaKoV could be frequently detected in younger dogs (25.45\%). Our result showed no seasonal pattern of CaKoV infection in domestic dogs. In this study, we characterized CaKoVs by whole genome sequencing $(n=4)$ or 3D and VP1 gene sequencing $(n=8)$. Genetic and phylogenetic analyses showed that whole genomes of Thai CaKoVs were closely related to Chinese CaKoVs with highest $99.5 \%$ amino acid identity suggesting possible origin of CaKoVs in Thailand.

Conclusions: In conclusion, this study was the first to report the detection and genetic characteristics of CaKoVs in domestic dogs in Thailand. CaKoVs could be detected in both sick and healthy dogs. The virus is frequently detected in younger dogs. Thai CaKoVs were genetically closely related and grouped with Chinese CaKoVs. Our result raises the concerns to vet practitioners that diarrhea in dogs due to canine Kobuvirus infection should not be ignored.
\end{abstract}

Keywords: Canine, Characterization, Detection, Kobuvirus, Thailand

\section{Background}

Kobuvirus (KoV) is a single-strand positive-sense RNA virus. KoV belongs to the family Piconaviridae, genus Kobuvirus, which consists of four species Aichivirus A, B, $\mathrm{C}$ and D [1-3]. KoV has been reported in feces of several mammal species including humans, ruminants, pigs, dogs, cats, bats and rodents [3-10]. The Kobuvirus species Aichivirus A contains four types including Aichi virus 1, canine Kobuvirus 1 (CaKoV), Feline Kobuvirus 1 (FeKoV)

\footnotetext{
* Correspondence: alongkorn.a@chula.ac.th

${ }^{1}$ Center of Excellence for Emerging and Re-emerging Infectious Diseases in

Animals, Faculty of Veterinary Science, Chulalongkorn University, Bangkok,

Thailand

${ }^{2}$ Department of Veterinary Public Health, Faculty of Veterinary Science,

Chulalongkorn University, Bangkok 10330, Thailand

Full list of author information is available at the end of the article
}

and Murine Kobuvirus 1 (MuKoV). Canine Kobuvirus 1 $(\mathrm{CaKoV})$ was first reported in dogs with acute gastroenteritis in the US in $2011[5,11]$. CaKoV was subsequently reported in dogs in UK, Italy, Australia, Japan, Korea and China $[4,12-15]$. The virus was reported in wild carnivores (Jackal and Hyena) and domestic dogs in Tanzania, Africa [16], in foxes in Spain [17] and in foxes [18] and wolves in Italy [19]. Several studies have reported the detection of $\mathrm{CaKoV}$ infection in dogs with or without diarrhea and sometime systemic infection [20]. To date, only 12 completed $\mathrm{CaKoV}$ genomes are available in the GenBank database.

During September 2016 to September 2018, the center of excellence for emerging and re-emerging infectious diseases in animals (CUEIDAs), Chulalongkorn University conducted

(c) The Author(s). 2019 Open Access This article is distributed under the terms of the Creative Commons Attribution 4.0 International License (http://creativecommons.org/licenses/by/4.0/), which permits unrestricted use, distribution, and reproduction in any medium, provided you give appropriate credit to the original author(s) and the source, provide a link to the Creative Commons license, and indicate if changes were made. The Creative Commons Public Domain Dedication waiver (http://creativecommons.org/publicdomain/zero/1.0/) applies to the data made available in this article, unless otherwise stated. 
a survey of canine Kobuvirus in domestic dogs at small animal hospitals in 5 provinces of Thailand. The survey was conducted under the Chulalongkorn University's animal use and care protocol \# 1731074. The result of this study provided the first detection and genetic characterization of $\mathrm{CaKoV}$ isolated from domestic dogs in Thailand.

\section{Results}

\section{Canine Kobuviruses in domestic dogs in Thailand}

During September 2016 to September 2018, we conducted a survey of viral enteric diseases in domestic dogs in small animal hospitals in 5 provinces of Thailand (Bangkok, Nakhon Ratchasima, Ratchaburi, Suphanburi, and Tak). We tested 307 rectal swab samples for $\mathrm{CaKoV}$ by using one-step RT-PCR specific to 3D gene. Based on a two-year survey, we found $\mathrm{CaKoV}$ positivity at $17.59 \%$ (54/307). CaKoVs could be detected in both sick $(19.44 \%(49 / 252))$ and healthy $(9.09 \%(5 / 55))$ animals. Our result showed no seasonal pattern of $\mathrm{CaKoV}$ infection in dogs (Figs. 1 and 2). In relation to age group, $\mathrm{CaKoV}$ could be frequently detected in younger dogs at 25.45\% (42/165) (Additional file 2: Table S2). The coinfections of $\mathrm{CaKoV}$ with other enteric viral pathogens were observed including $\mathrm{CaKoV} / \mathrm{Canine}$ parvovirus/Canine Coronavirus $(n=6), \mathrm{CaKoV} / \mathrm{Canine}$ parvovirus $(n=20)$ and $\mathrm{CaKoV} /$ Canine Coronavirus $(n=2)$. In this study, 12 $\mathrm{CaKoVs}$ were selected and characterized by whole genome sequencing $(n=4)$ or $3 \mathrm{D}$ and VP1 gene sequencing $(n=8)$. The viruses were selected to represent epidemiological and demographic data such as age, date of isolation and breed. In this study, nucleotide sequences of the $\mathrm{CaKoV}$ were submitted to the GenBank database under the accession numbers MK201776 - MK201795 (Table 1).

\section{Phylogeny of the Thai canine Kobuviruses}

Phylogenetic analysis of whole genome of CaKoVs showed that the Thai CaKoVs were closely related to each other and clustered with Aichivirus A. The cluster Aichivirus A contains Kobuviruses from dogs, cats, rodents, bats and human. While Aichivirus B and C contain Kobuviruses from cattle and pigs, respectively. Based on whole genome sequence, Thai CaKoVs were closely related to Chinese CaKoVs sub-cluster but in separated sub-cluster from the viruses from the US, UK, Brazil and Tanzania (Fig. 3). Phylogenetic analysis of 3D and VP1 of Thai CaKoVs and reference CaKoVs from various animal species were also performed. Similarly, 3D gene of Thai CaKoVs were grouped together with Chinese CaKoVs (G1 sub-cluster) but separated from the viruses in sub-clusters G2 as well as G3 (Fig. 4). Phylogenetic analysis of VP1 gene, the viruses can be clustered into 2 major subgroups, US/EU/Africa subgroup and China/Thailand subgroup (Fig. 5).

\section{Genetic analysis of the Thai canine Kobuviruses}

We compared the nucleotide and deduced amino acid sequences of Thai CaKoVs against those of reference viruses from the US, UK, Italy, China, and Korea (Tables 2 and 3). Our results showed that whole genome of 4 Thai CaKoVs (CU-53, CU-101, CU-249 and CU-716) shared 96.7-99.3\% nucleotide similarity (99.6-100\% amino acid similarity) to each other and posed highest nucleotide similarity to Chinese CaKoVs including SMCD-59 (97.0\% nt and 99.5\% aa identity) and $\mathrm{CH}-1$ ( $96.8 \%$ nt and $98.7 \%$ aa identity). Our analysis showed that the VP1 protein was the most diverse gene with 93.4-99.9\% nucleotide similarity (96.9-100\% aa similarity) among Thai CaKoVs and $82.2-96.8 \%$ with other reference CaKoVs. The

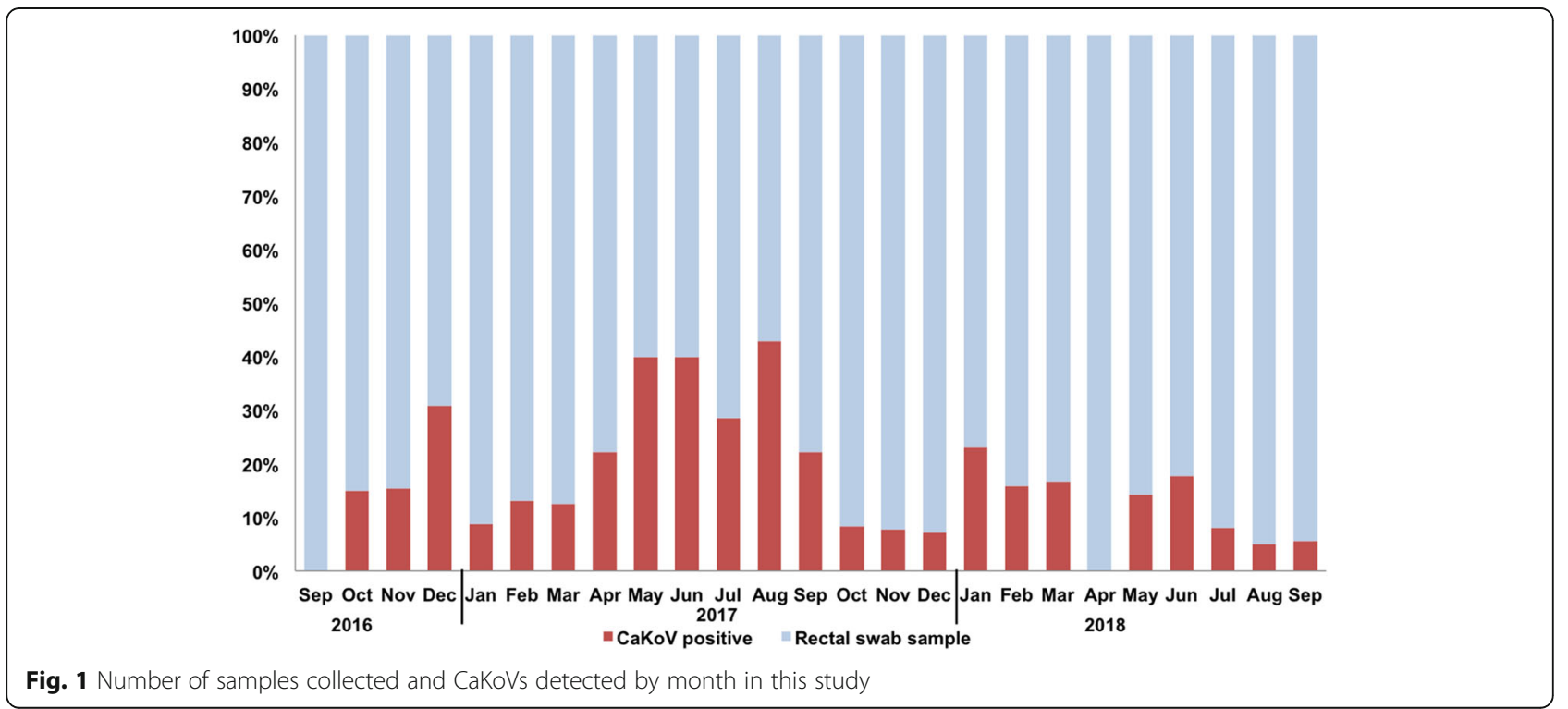




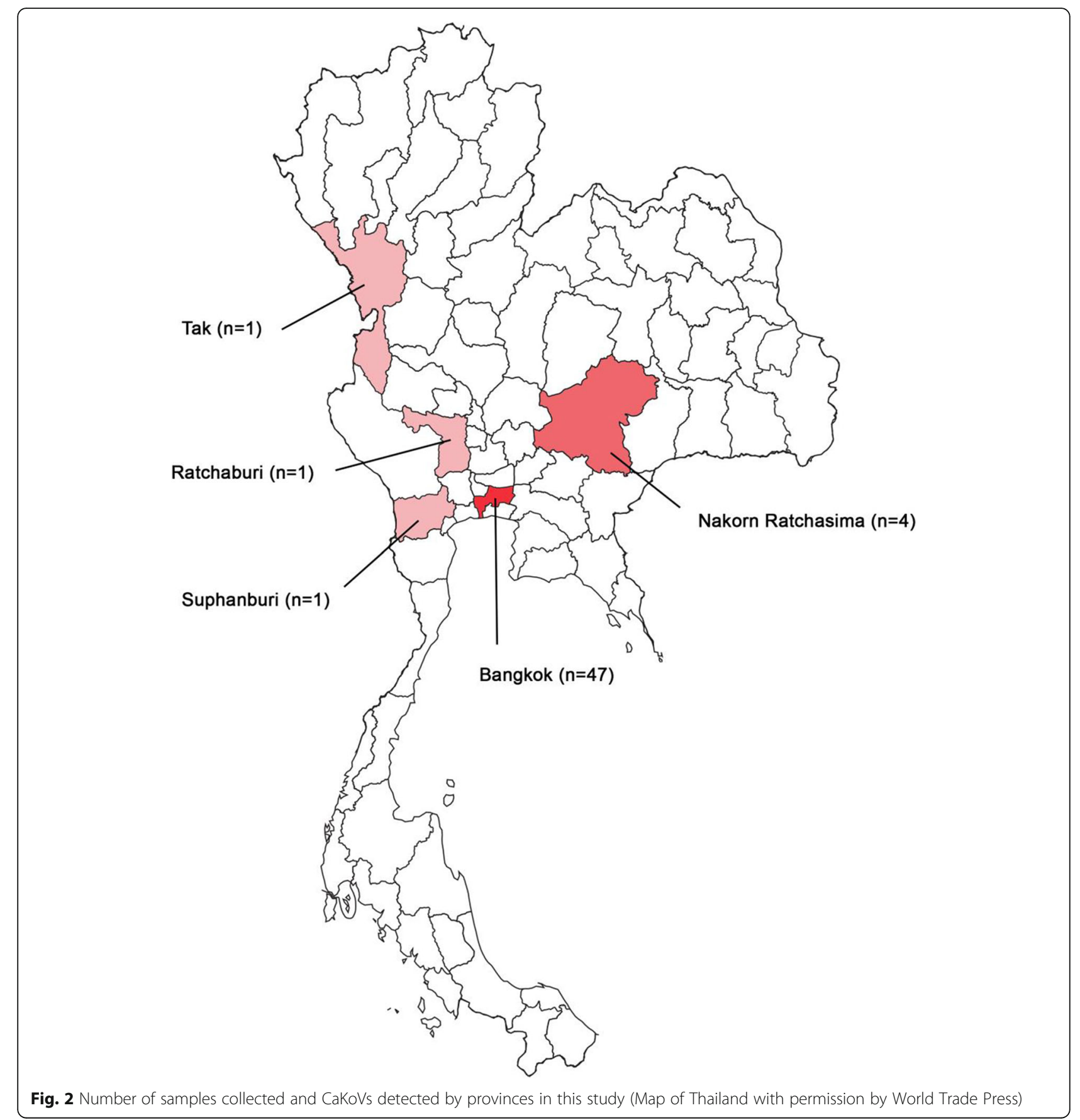

most variable region of VP1 is position 201-243, especially proline rich region. Putative proline rich region at VP1-228-240 ( $\left.\mathrm{P}_{228} \mathrm{XPPPPXPPXPXP}{ }_{240}\right)$ was also observed in Thai CaKoVs as well as reference viruses (Table 4). In this study, unique amino acids were found in Thai and Chinese CaKoVs at the position, $65 \mathrm{~V}, 67 \mathrm{D}, 119 \mathrm{~L}, 138 \mathrm{~T}, 150 \mathrm{P}, 151 \mathrm{M}, 153 \mathrm{D}$, 201S, 204Q, 205Q, 201Q, 213T and 241E (Table 4). Analysis of predicted amino acid cleavage sits of whole genome were conserved among Thai CaKoVs (Table 5).

\section{Discussions}

Canine Kobuvirus $(\mathrm{CaKoV})$ is an emerging pathogen in Thailand. To the best of our knowledge, the $\mathrm{CaKoV}$ was described in Asia in retrospective study in Korea in 2011 and have been reported in Japan, China and Australia, respectively $[2,15,17,21]$. However, the $\mathrm{CaKoV}$ have 
Table 1 Detail description of Thai CaKoVs characterized in this study

\begin{tabular}{|c|c|c|c|c|c|c|c|c|}
\hline Virus & Date & Location & Region & Age & Breed & Clinical signs & Sequencing & $\begin{array}{l}\text { GenBank } \\
\text { Accession number }\end{array}$ \\
\hline CU-53 & Oct-16 & Bangkok & Central & 2 months & Pomeranian & Diarrhea & $W G^{a}$ & MK201776 \\
\hline CU-101 & Dec-16 & Bangkok & Central & 3 months & Pekingese & Diarrhea & WG & MK201777 \\
\hline CU-249 & May-17 & Bangkok & Central & 3 months & Pomeranian & Diarrhea & WG & MK201778 \\
\hline CU-716 & Jan-18 & Bangkok & Central & 12 years & Shizu & Diarrhea & WG & MK201779 \\
\hline CU-83 & Nov-16 & Bangkok & Central & 2 months & Pomeranian & Diarrhea & $3 \mathrm{D}, \mathrm{VP} 1^{\mathrm{b}}$ & MK201780, MK201788 \\
\hline CU-100 & Dec-16 & Ratchaburi & Central & 6 months & Great Dane & Diarrhea & 3D, VP1 & MK201781, MK20178s \\
\hline CU-125 & Jan-17 & Tak & Northern & 2 months & Bang Keaw & Asymptomatic & 3D, VP1 & MK201782, MK201790 \\
\hline CU-224 & Feb-17 & Bangkok & Central & 9 years & Pomeranian & Diarrhea & 3D, VP1 & MK201783, MK201791 \\
\hline CU-241 & Apr-17 & Bangkok & Central & 3 months & Mixed & Diarrhea & 3D, VP1 & MK201784, MK201792 \\
\hline CU-250 & May-17 & Bangkok & Central & 3 months & Pomeranian & Diarrhea & 3D, VP1 & MK201785, MK201793 \\
\hline CU-260 & Jun-17 & Nakhon Ratchasima & North- Eastern & 2 months & German Shepherd & Diarrhea & 3D, VP1 & MK201786, MK201794 \\
\hline CU-273 & Aug-17 & Bangkok & Central & 2 months & Pomeranian & Diarrhea & 3D, VP1 & MK201787, MK20179 \\
\hline
\end{tabular}

${ }^{a} W G$ Whole genome sequencing

b 3D, VP1: 3D and VP1 gene sequencing

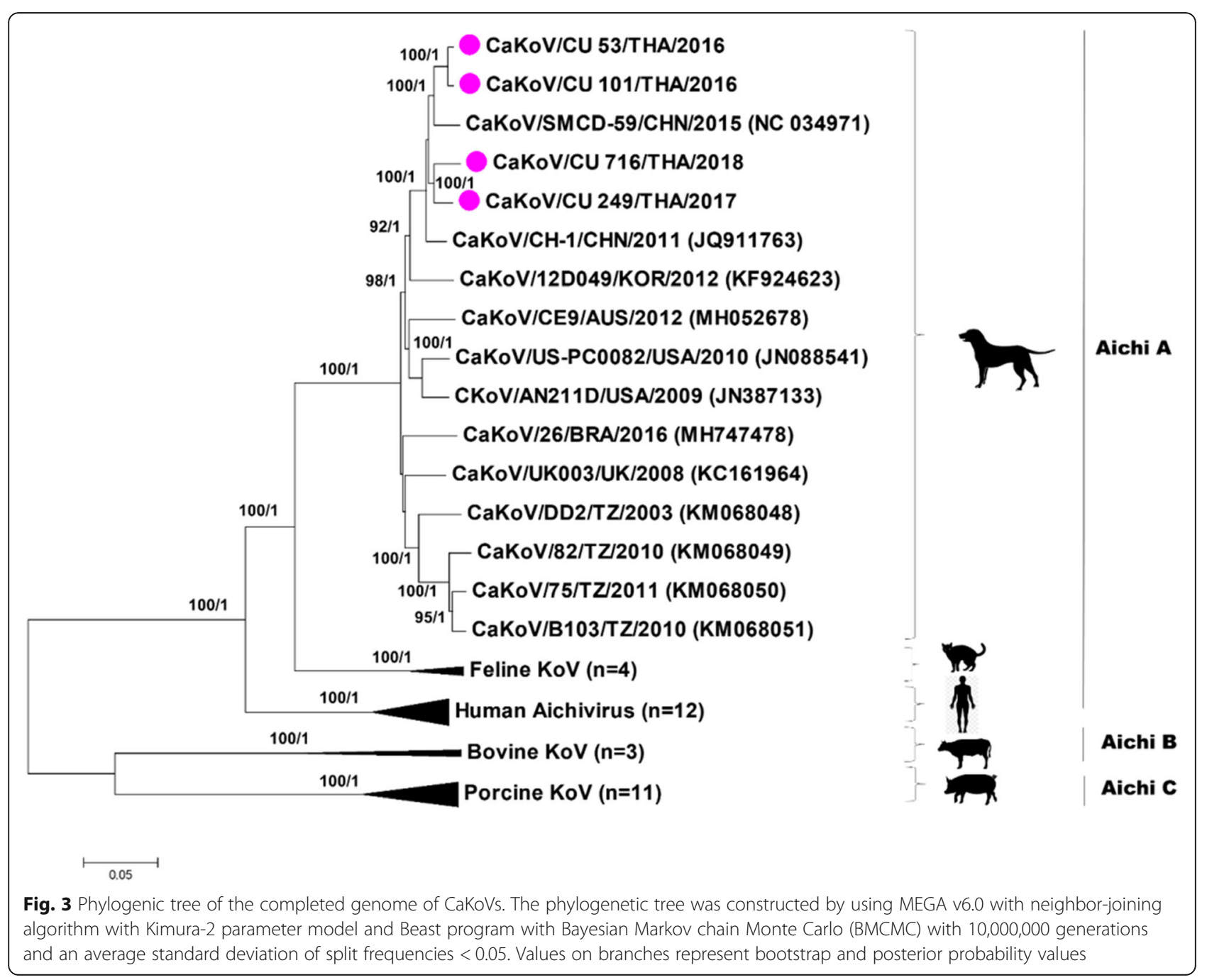




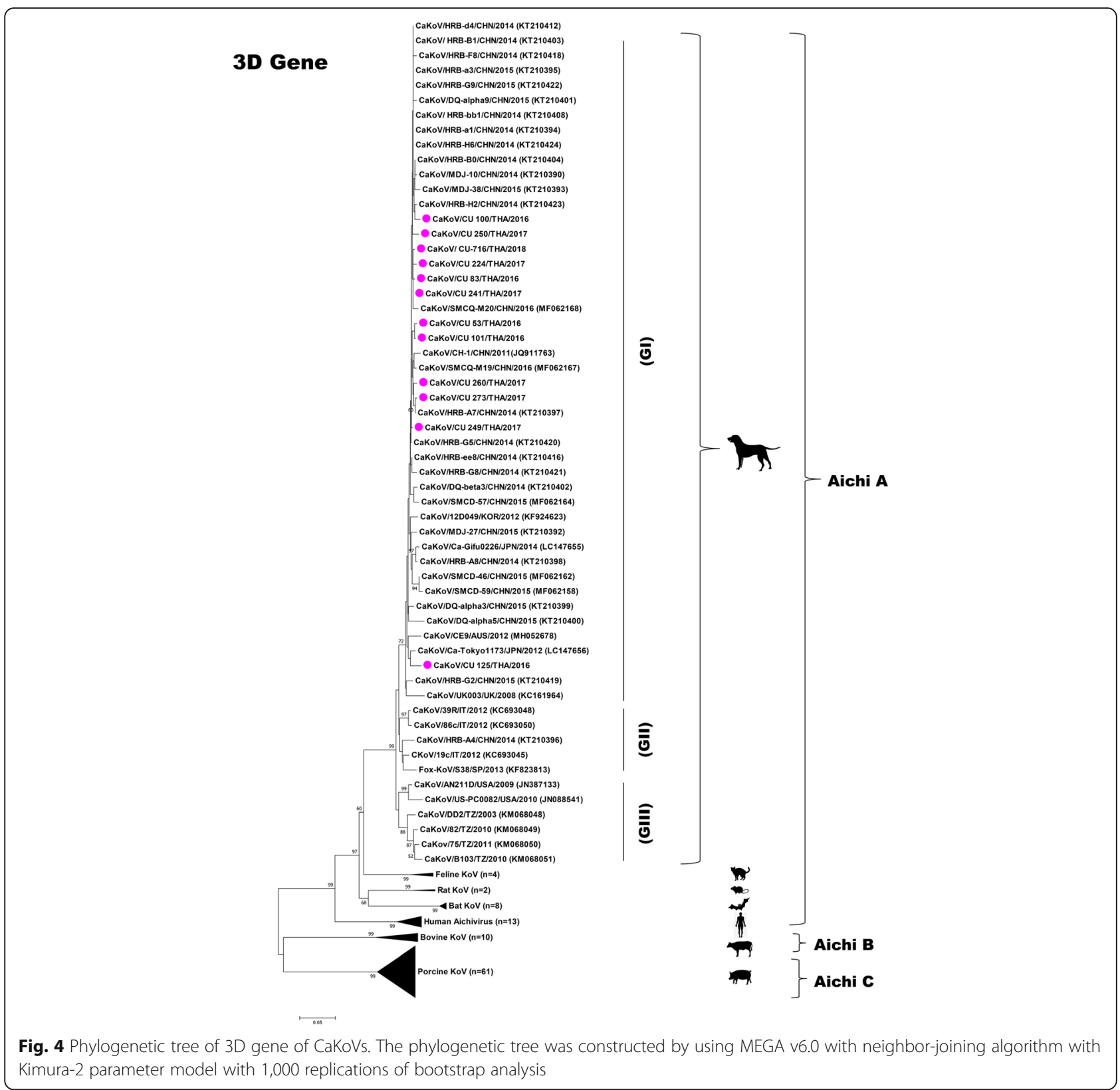

never been reported in the country or South East Asia region. In this study, during the 2 year-survey program, we found $\mathrm{CaKoV}$ positivity at $17.59 \%$ in both sick (19.44\%) and healthy (9.09\%) animals. Compare to other studies, $\mathrm{CaKoV} \%$ positivity in this study was lower than those in China (54\%) and Korea (32.2\%) [14, 22]. Our result showed that the $\mathrm{CaKoV}$ could be frequently detected in younger dogs at $27 \%$ which consistence with previous reports [15]. Similar to other previous studies, co-infections with other enteric viral pathogens were observed such as $\mathrm{CaKoV} / \mathrm{Canine}$ parvovirus and $\mathrm{CaKoV} /$ Canine Coronavirus $[12,14,15]$. Moreover, CaKoVs were detected in both diarrheic and non-diarrheic dogs which consistent with other studies $[2,15]$. Our result supported that this virus may not be the only cause of enteric disease in dogs. Nevertheless, the CaKoV infection have still been identified in symptomatic dogs without other enteric pathogen infections [12]. Our observation supported that the role of $\mathrm{CaKoV}$ as a primary pathogen of acute gastroenteritis remain unclear.

In this study, the genome size of 4 Thai CaKoVs is 7 , $530 \mathrm{bp}$ with one ORF encoding 2,444 amino acids of a putative polyprotein, which comparable to previous reports. Genome organization of $\mathrm{CaKoV}$ includes leader protein (L), structural proteins (VP0, VP3, VP1), nonstructural proteins $(2 \mathrm{~A}, 2 \mathrm{~B}, 2 \mathrm{C}, 3 \mathrm{~A}, 3 \mathrm{~B}, 3 \mathrm{C}, 3 \mathrm{D})$. 


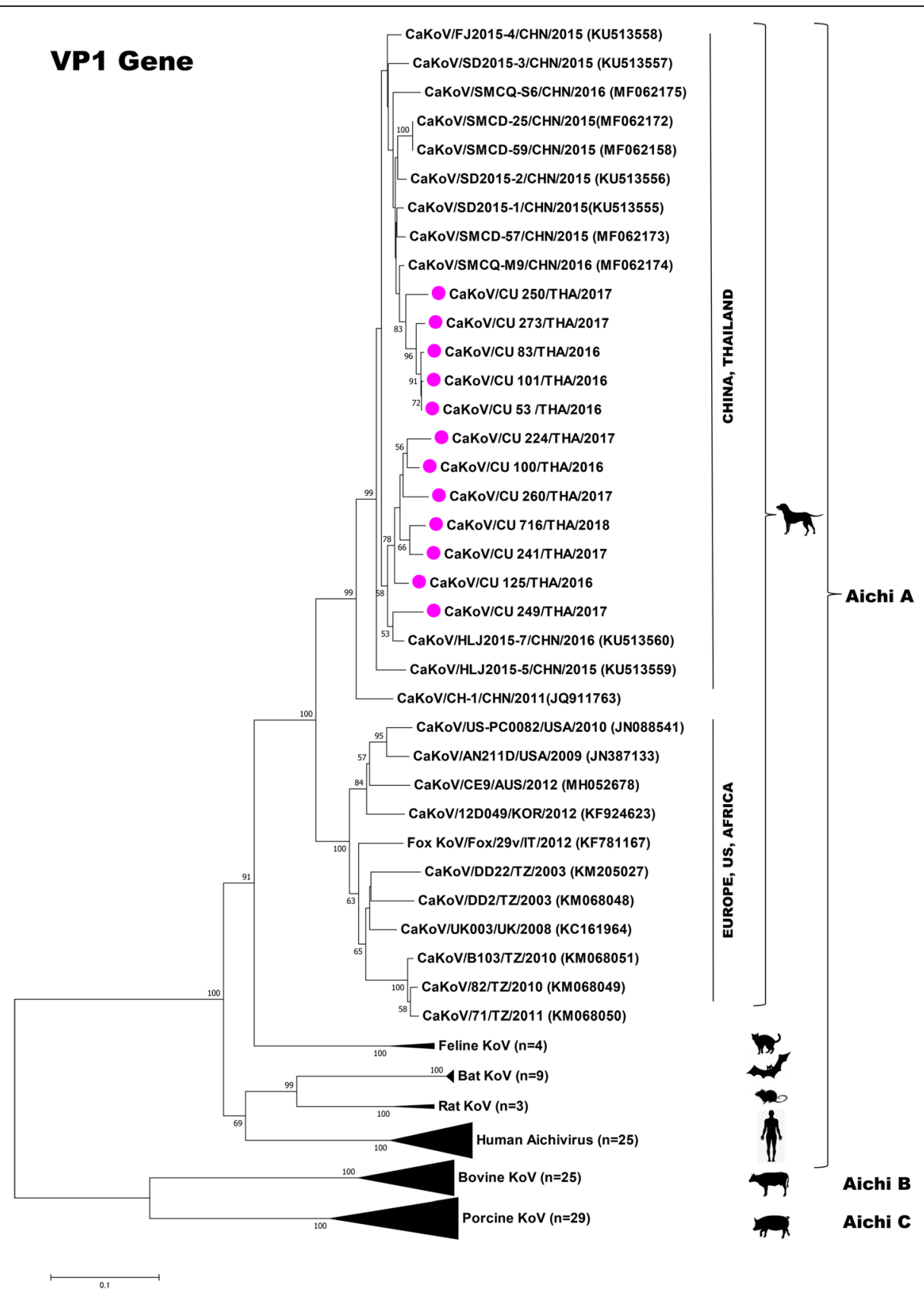

Fig. 5 Phylogenetic tree of VP1 gene of CaKoVs. The phylogenetic tree was constructed by using MEGA v6.0 with neighbor-joining algorithm with Kimura-2 parameter model with 1,000 replications of bootstrap analysis

Phylogenetic analyses showed that the Thai CaKoVs were closely related to each other and clustered with Aichivirus A. It is noted that Thai CaKoVs were closely related to Chinese CaKoVs sub-cluster but in separated sub-cluster from the viruses from the US, UK, Brazil and Tanzania (Fig. 3). Phylogenetic analyses of 3D gene showed similar result which Thai CaKoVs were grouped together with Chinese CaKoVs (G1 sub-cluster). This observation regarding to the sub-clusters of CaKoVs was in agreement with the previous study [23]. On the other hand, based on VP1 gene, the viruses can be clustered into 2 major subgroups, US/EU/Africa subgroup and China/Thailand subgroup which similar to the previous reports [16, 22] (Figs. 4 and 5).

Genetic analyses of Thai CaKoVs showed that whole genome of 4 Thai CaKoVs posed highest nucleotide similarity to Chinese CaKoVs including SMCD-59 and $\mathrm{CH}-1$. This observation supported phylogenetic analysis that Thai CaKoVs were closely related to Chinese $\mathrm{CaKoVs}$ sub-cluster but in separated sub-cluster from 


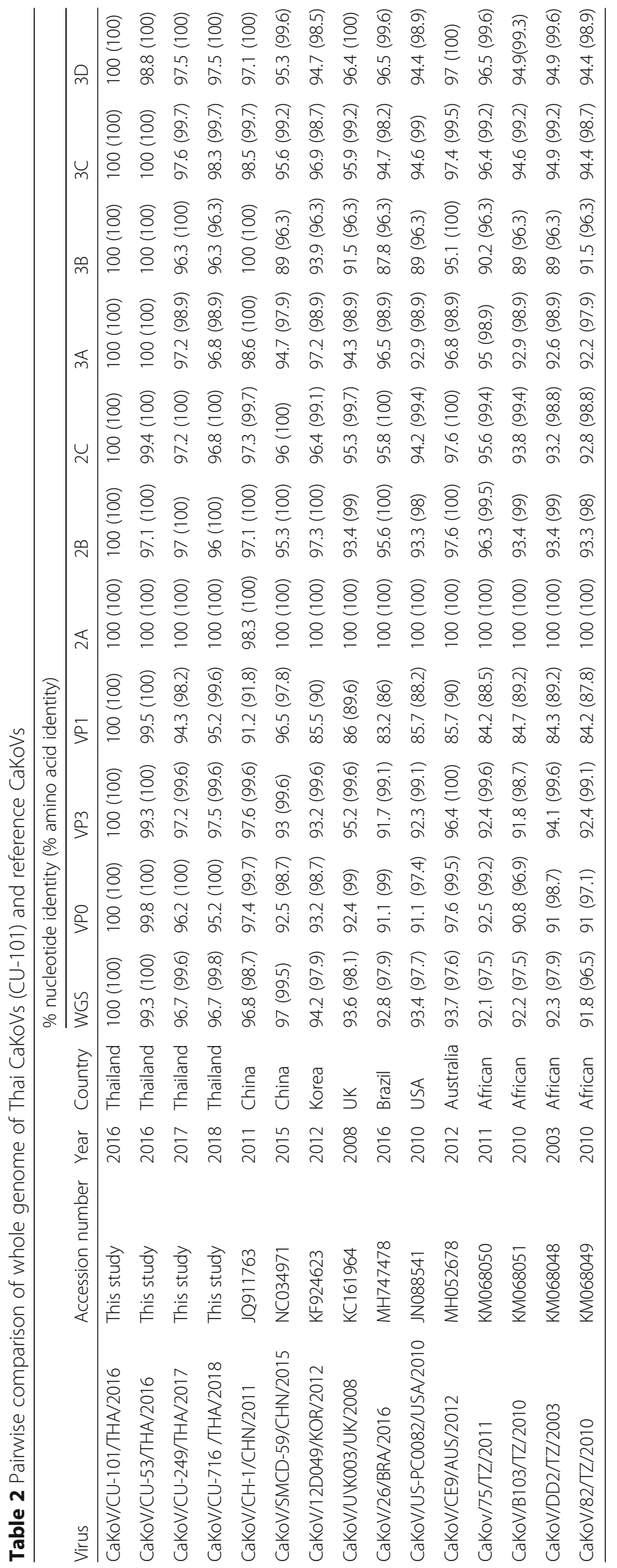


Table 3 Pairwise comparison of 3D and VP1 genes of Thai CaKoVs (CU-101) and reference CaKoVs

\begin{tabular}{|c|c|c|c|c|c|}
\hline \multirow[t]{2}{*}{ Viruses } & \multirow{2}{*}{$\begin{array}{l}\text { Accession } \\
\text { number }\end{array}$} & \multirow[t]{2}{*}{ Year } & \multirow[t]{2}{*}{ Country } & \multicolumn{2}{|c|}{$\%$ nucleotide identity (\% amino acid identity) } \\
\hline & & & & $3 \mathrm{D}$ & VP1 \\
\hline CaKoV/CU-101/THA/2016 & This study & 2016 & Thailand & $100(100)$ & $100(100)$ \\
\hline CaKoV/CU-53/THA/2016 & This study & 2016 & Thailand & $99.5(100)$ & $99.9(100)$ \\
\hline CaKoV/CU-83/THA/2016 & This study & 2016 & Thailand & $98.8(100)$ & $99.7(100)$ \\
\hline CaKoV/CU-100/THA/2016 & This study & 2016 & Thailand & $97.9(100)$ & $93.6(97.8)$ \\
\hline CaKoV/CU-125/THA/2016 & This study & 2016 & Thailand & $97.1(98.6)$ & $94.9(97.8)$ \\
\hline CaKoV/CU-224/THA/2017 & This study & 2017 & Thailand & $98.6(100)$ & $93.6(97.8)$ \\
\hline CaKoV/CU-241/THA/2017 & This study & 2017 & Thailand & $99.0(100)$ & $94.5(98.7)$ \\
\hline CaKoV/CU-249/THA/2017 & This study & 2017 & Thailand & $98.8(100)$ & $93.6(97.4)$ \\
\hline CaKoV/CU-250/THA/2017 & This study & 2017 & Thailand & $98.1(100)$ & $96.6(96.9)$ \\
\hline CaKoV/CU-260/THA/2017 & This study & 2017 & Thailand & $98.6(100)$ & $93.4(96.9)$ \\
\hline CaKoV/CU-273/THA/2017 & This study & 2017 & Thailand & $98.6(100)$ & $98.5(99.1)$ \\
\hline CaKoV/CU-716/THA/2018 & This study & 2018 & Thailand & $98.8(100)$ & $94.3(98.7)$ \\
\hline CaKoV/26/BRA/2016 & MH747478 & 2016 & Brazil & $97.1(99.3)$ & $82.2(84.2)$ \\
\hline CaKoV/CE9/AUS/2012 & MH052678 & 2012 & Australia & $97.6(100)$ & $83.7(87.7)$ \\
\hline CaKoV/B103/TZ/2010 & KM068051 & 2010 & African & 93.6 (98.6) & $84.8(88.2)$ \\
\hline CaKov/75/TZ/2011 & KM068050 & 2011 & African & $94.0(97.9)$ & $83.8(86.4)$ \\
\hline CaKoV/82/TZ/2010 & KM068049 & 2010 & African & 94.5 (98.6) & $84.3(86.4)$ \\
\hline CaKoV/DD2/TZ/2003 & KM068048 & 2003 & African & 94.8 (99.3) & $84.0(87.7)$ \\
\hline CaKoV/UK003/UK/2008 & KC161964 & 2008 & UK & $96.0(100)$ & $85.3(88.2)$ \\
\hline CaKoV/US-PC0082/USA/2010 & JN088541 & 2010 & USA & $94.0(99.3)$ & $84.5(86.4)$ \\
\hline CaKoV/AN211D/USA/2009 & JN387133 & 2009 & USA & $95.2(99.3)$ & $84.4(86.8)$ \\
\hline CaKoV/86c/IT/2012 & KC693050 & 2012 & Italy & $96.0(99.3)$ & $\mathrm{N} / \mathrm{A}$ \\
\hline CKoV/19C/IT/2012 & KC693045 & 2012 & Italy & $96.2(99.3)$ & $\mathrm{N} / \mathrm{A}$ \\
\hline CaKoV/Ca-Gifu0226/JPN/2014 & LC147655 & 2014 & Japan & $97.6(99.3)$ & $\mathrm{N} / \mathrm{A}$ \\
\hline CaKoV/Ca-Tokyo1173/JPN/2012 & LC147656 & 2012 & Japan & $97.9(100)$ & $\mathrm{N} / \mathrm{A}$ \\
\hline CaKoV/12D049/KOR/2012 & KF924623 & 2012 & Korea & $97.1(100)$ & $84.7(89.0)$ \\
\hline CaKoV/CH-1/CHN/2011 & JQ911763 & 2016 & China & $97.9(100)$ & $91.3(89.9)$ \\
\hline CaKoV/SMCD-59/CHN/2015 & MF062158 & 2015 & China & $97.1(100)$ & $96.4(96.9)$ \\
\hline CaKoV/SMCD-57/CHN/2015 & MF062173 & 2015 & China & $97.9(100)$ & $96.8(97.8)$ \\
\hline
\end{tabular}

the viruses from the US, UK, Brazil and Tanzania. Of all viral genes, the VP1 gene was the most diverse gene among Thai CaKoVs and other reference CaKoVs. Similar observation was also reported in previous study that VP1 protein is the most variable capsid protein [24]. It is noted that the putative proline rich region at VP1-228$240\left(\mathrm{P}_{228} \mathrm{XPPPPXPPXPXP}{ }_{240}\right)$ was observed both in Thai $\mathrm{CaKoVs}$ and reference viruses. Previous studies indicated that proline rich region may associate with enteric receptor binding of the viruses [14, 24]. It is noted that Thai CaKoVs posed unique PPP (VP1; 228-240), which also observed most reference viruses from China, Korea, Japan, US, UK suggesting unique characteristic. These unique amino acids were not observed in the $\mathrm{CaKoV}$ from the Australia (CE9), Brazil (BRA/26) and Tanzania (TZ/75, TZ82) [16, 20]. However, the association of these unique amino acids and viral pathogenesis is still need to be further investigated. Based on genetic analysis, unique amino acids at the position, $65 \mathrm{~V}, 67 \mathrm{D}, 119 \mathrm{~L}, 138$ T, 150P, 151M, 153D, 201S, 204Q, 205Q, 201Q, $213 \mathrm{~T}$ and $241 \mathrm{E}$ were observed. These unique amino acids of China/ Thailand sub-cluster could be benefit for the detection of virus origin or diagnostic purpose in the future. Similar to previous study, analysis of predicted amino acid cleavage sits of whole genome were conserved among CaKoVs except one variation at 776/777 (VP3/VP1) which unique in wild carnivores [16].

\section{Conclusions}

In conclusion, this study is the first to report of canine Kobuvirus in dogs in Thailand. CaKoVs were mostly detected in clinical dogs of young age. However, the viruses 
Table 4 Genetic analysis of Thai CaKoVs compared with reference CaKoVs at proline rich region

\begin{tabular}{|c|c|c|c|c|c|c|c|c|c|c|c|c|c|c|c|c|c|}
\hline \multirow[t]{2}{*}{ Viruses } & \multirow{2}{*}{$\begin{array}{l}\text { Accession } \\
\text { number }\end{array}$} & \multirow[t]{2}{*}{ Year } & \multirow[t]{2}{*}{ Country } & \multicolumn{14}{|c|}{ Amino acid at position } \\
\hline & & & & 65 & 67 & 119 & 138 & 150 & 151 & 153 & 201 & 204 & 205 & 210 & 213 & 241 & $\begin{array}{l}\text { Proline rich region } \\
(228-240)\end{array}$ \\
\hline CaKoV/CU-101/THA/2016 & This study & 2016 & Thailand & V & $\mathrm{D}$ & $\mathrm{L}$ & $\mathrm{T}$ & $P$ & M & D & $S$ & Q & Q & Q & $\mathrm{T}$ & $E$ & PRAPPPLPPLPTP \\
\hline CaKoV/CU-53/THA/2016 & This study & 2016 & Thailand & V & $\mathrm{D}$ & L & $\mathrm{T}$ & $P$ & M & D & S & Q & Q & Q & $\mathrm{T}$ & E & PRAPPPLPPLPTP \\
\hline CaKoV/CU-249/THA/2017 & This study & 2017 & Thailand & V & $\mathrm{D}$ & L & $\mathrm{T}$ & $P$ & M & D & S & Q & Q & Q & $\mathrm{T}$ & E & PRAPPPLPPLPTP \\
\hline CaKoV/CU-716/THA/2018 & This study & 2018 & Thailand & V & $\mathrm{D}$ & L & $\mathrm{T}$ & $P$ & M & D & S & Q & Q & Q & $\mathrm{T}$ & E & PRAPPPLPPLPTP \\
\hline CaKoV/SMCQ-M9/CHN/2016 & MF062174 & 2016 & China & V & $D$ & L & $\mathrm{T}$ & $P$ & M & D & S & Q & Q & Q & $\mathrm{T}$ & $\mathrm{E}$ & PRAPPPLPPLPTP \\
\hline CaKoV/SMCD-59/CHN/2015 & NC 034971 & 2015 & China & V & $\mathrm{D}$ & L & $\mathrm{T}$ & $P$ & M & $\mathrm{D}$ & S & Q & Q & Q & $\mathrm{T}$ & E & PRAPPPLPPLPTP \\
\hline CaKoV/12D049/KOR/2012 & KF924623 & 2012 & Korea & L & $\mathrm{N}$ & V & M & S & E & $\mathrm{N}$ & $\mathrm{T}$ & V & $\mathrm{E}$ & S & S & A & PRAPPPLPPLPTP \\
\hline CaKoV/CE9/AUS/2012 & MH052678 & 2012 & Australia & L & $\mathrm{N}$ & V & M & S & E & $\mathrm{N}$ & $\mathrm{T}$ & V & E & S & S & T & PRAPP-L_PPLPTP \\
\hline CaKoV/AN211D/USA/2009 & JN387133 & 2009 & USA & L & N & $P$ & M & S & $\mathrm{E}$ & $\mathrm{N}$ & $\mathrm{T}$ & V & $\mathrm{E}$ & S & S & A & PRAPPPLPPLPTP \\
\hline CaKoV/US-PC0082/USA/2010 & JN088541 & 2010 & USA & L & N & V & M & S & E & $\mathrm{N}$ & $\mathrm{T}$ & V & E & S & S & A & $\underline{\text { CPVPPPLPPLPTP }}$ \\
\hline CaKoV/UK003/UK/2008 & KC161964 & 2008 & UK & L & N & V & M & $S$ & E & $\mathrm{N}$ & $\mathrm{T}$ & V & $\mathrm{E}$ & S & S & T & PRAPPPLPPLPTP \\
\hline CaKoV/26/BRA/2016 & MH747478 & 2016 & Brazil & L & $\mathrm{N}$ & V & M & S & E & $\mathrm{N}$ & $\mathrm{T}$ & V & $\mathrm{E}$ & S & S & T & $\underline{\text { HGAPPPLPPLPTP }}$ \\
\hline CaKoV/75/TZ/2011 & KM068050 & 2011 & Africa & L & N & V & M & S & E & $\mathrm{N}$ & $\mathrm{T}$ & A & $E$ & S & S & T & CPVPPPLPPLPTP \\
\hline CaKoV/82/TZ/2010 & KM068049 & 2010 & Africa & L & $\mathrm{N}$ & V & M & $S$ & E & $\mathrm{N}$ & $\mathrm{T}$ & A & $E$ & $S$ & S & T & $\underline{\text { CPVPPPLPPLPTP }}$ \\
\hline CaKoV/B103/TZ/2010 & KM068051 & 2010 & Africa & L & N & V & M & S & E & $\mathrm{N}$ & $\mathrm{T}$ & A & $E$ & S & S & T & PRAPPPLPPLPTP \\
\hline CaKoV/DD2/TZ/2003 & KM068048 & 2003 & Africa & L & $\mathrm{N}$ & V & $M$ & S & E & N & $\mathrm{T}$ & V & $\mathrm{E}$ & S & S & T & PRAPPPLPPLPTP \\
\hline
\end{tabular}

could be detected from both healthy and sicked dogs. Genetic and phylogenetic analyses showed that whole genomes of Thai CaKoVs were closely related to Chinese CaKoVs in 2015 (SMCD-59) with high nucleotide similarity suggesting a possible origin of $\mathrm{CaKoVs}$ in Thailand. $\mathrm{CaKoV}$ is considered as an emerging viral pathogen in the domestic dogs. Since CaKoVs have never been reported in the country and SEA region, the detection and characterization of $\mathrm{CaKoV}$ from different parts of the regions should be extended for better understanding the epidemiology and evolution of CaKoVs. Our result raises the concerns to vet practitioners that diarrhea in dogs due to canine Kobuvirus infection should not be ignored.

Table 5 Genetic analysis of Thai CaKoVs compared with reference CaKoVs at putative amino acid cleavage sites

\begin{tabular}{|c|c|c|c|c|c|c|c|c|c|c|c|c|}
\hline \multirow[t]{2}{*}{ Viruses } & \multirow[t]{2}{*}{ Year } & \multirow[t]{2}{*}{ Country } & \multicolumn{10}{|c|}{ Amino acid position } \\
\hline & & & $171 / 172$ & $553 / 554$ & 776/777 & $1054 / 1055$ & $1165 / 1166$ & 1330/1331 & $1665 / 1666$ & $1759 / 1760$ & $1786 / 1787$ & $2176 / 2177$ \\
\hline CU-53 & 2016 & Thailand & $\mathrm{Q} / \mathrm{G}$ & $\mathrm{Q} / \mathrm{H}$ & $\mathrm{Q} / \mathrm{A}$ & YN & $\mathrm{Q} / \mathrm{G}$ & $\mathrm{Q} / \mathrm{G}$ & $\mathrm{Q} / \mathrm{G}$ & $\mathrm{Q} / \mathrm{A}$ & $\mathrm{Q} / \mathrm{G}$ & $\mathrm{Q} / \mathrm{G}$ \\
\hline CU-101 & 2016 & Thailand & $\mathrm{Q} / \mathrm{G}$ & $\mathrm{Q} / \mathrm{H}$ & $\mathrm{Q} / \mathrm{A}$ & YN & $\mathrm{Q} / \mathrm{G}$ & $\mathrm{Q} / \mathrm{G}$ & $\mathrm{Q} / \mathrm{G}$ & $\mathrm{Q} / \mathrm{A}$ & $\mathrm{Q} / \mathrm{G}$ & $\mathrm{Q} / \mathrm{G}$ \\
\hline CU-249 & 2017 & Thailand & $\mathrm{Q} / \mathrm{G}$ & $\mathrm{Q} / \mathrm{H}$ & $\mathrm{Q} / \mathrm{A}$ & YN & $\mathrm{Q} / \mathrm{G}$ & $\mathrm{Q} / \mathrm{G}$ & $\mathrm{Q} / \mathrm{G}$ & $\mathrm{Q} / \mathrm{A}$ & $\mathrm{Q} / \mathrm{G}$ & $\mathrm{Q} / \mathrm{G}$ \\
\hline CU-716 & 2018 & Thailand & $\mathrm{Q} / \mathrm{G}$ & $\mathrm{Q} / \mathrm{H}$ & $\mathrm{Q} / \mathrm{A}$ & YN & $\mathrm{Q} / \mathrm{G}$ & $\mathrm{Q} / \mathrm{G}$ & $\mathrm{Q} / \mathrm{G}$ & $\mathrm{Q} / \mathrm{A}$ & $\mathrm{Q} / \mathrm{G}$ & $\mathrm{Q} / \mathrm{G}$ \\
\hline 12D049 & 2012 & Korea & $\mathrm{Q} / \mathrm{G}$ & $\mathrm{Q} / \mathrm{H}$ & $\mathrm{Q} / \mathrm{A}$ & YN & $\mathrm{Q} / \mathrm{G}$ & $\mathrm{Q} / \mathrm{G}$ & $\mathrm{Q} / \mathrm{G}$ & $\mathrm{Q} / \mathrm{A}$ & $\mathrm{Q} / \mathrm{G}$ & $\mathrm{Q} / \mathrm{G}$ \\
\hline UK003 & 2008 & UK & $\mathrm{Q} / \mathrm{G}$ & $\mathrm{Q} / \mathrm{H}$ & $\mathrm{Q} / \mathrm{A}$ & YN & $\mathrm{Q} / \mathrm{G}$ & $\mathrm{Q} / \mathrm{G}$ & $\mathrm{Q} / \mathrm{G}$ & $\mathrm{Q} / \mathrm{A}$ & $\mathrm{Q} / \mathrm{G}$ & $\mathrm{Q} / \mathrm{G}$ \\
\hline 26/BRA & 2016 & Brazil & $\mathrm{Q} / \mathrm{G}$ & $\mathrm{Q} / \mathrm{H}$ & $\mathrm{Q} / \mathrm{A}$ & YN & $\mathrm{Q} / \mathrm{G}$ & $\mathrm{Q} / \mathrm{G}$ & $\mathrm{Q} / \mathrm{G}$ & $\mathrm{Q} / \mathrm{A}$ & $\mathrm{Q} / \mathrm{G}$ & $\mathrm{Q} / \mathrm{G}$ \\
\hline SMCD-59 & 2015 & China & $\mathrm{Q} / \mathrm{G}$ & $\mathrm{Q} / \mathrm{H}$ & $\mathrm{Q} / \mathrm{A}$ & YN & $\mathrm{Q} / \mathrm{G}$ & $\mathrm{Q} / \mathrm{G}$ & $\mathrm{Q} / \mathrm{G}$ & $\mathrm{Q} / \mathrm{A}$ & $\mathrm{Q} / \mathrm{G}$ & $\mathrm{Q} / \mathrm{G}$ \\
\hline CE9 & 2012 & Australia & $\mathrm{Q} / \mathrm{G}$ & $\mathrm{Q} / \mathrm{H}$ & $\mathrm{Q} / \mathrm{A}$ & YN & $\mathrm{Q} / \mathrm{G}$ & $\mathrm{Q} / \mathrm{G}$ & $\mathrm{Q} / \mathrm{G}$ & $\mathrm{Q} / \mathrm{A}$ & $\mathrm{Q} / \mathrm{G}$ & $\mathrm{Q} / \mathrm{G}$ \\
\hline B103 & 2010 & Africa & $\mathrm{Q} / \mathrm{G}$ & $\mathrm{Q} / \mathrm{H}$ & $\underline{\mathrm{Q} / \mathrm{T}^{\mathrm{a}}}$ & YN & $\mathrm{Q} / \mathrm{G}$ & $\mathrm{Q} / \mathrm{G}$ & $\mathrm{Q} / \mathrm{G}$ & $\mathrm{Q} / \mathrm{A}$ & $\mathrm{Q} / \mathrm{G}$ & $\mathrm{Q} / \mathrm{G}$ \\
\hline 75 & 2011 & Africa & $\mathrm{Q} / \mathrm{G}$ & $\mathrm{Q} / \mathrm{H}$ & $\mathrm{Q} / \mathrm{A}$ & YN & $\mathrm{Q} / \mathrm{G}$ & $\mathrm{Q} / \mathrm{G}$ & $\mathrm{Q} / \mathrm{G}$ & $\mathrm{Q} / \mathrm{A}$ & $\mathrm{Q} / \mathrm{G}$ & $\mathrm{Q} / \mathrm{G}$ \\
\hline 82 & 2010 & Africa & $\mathrm{Q} / \mathrm{G}$ & $\mathrm{Q} / \mathrm{H}$ & ${\underline{\mathrm{Q} / \mathrm{T}^{\mathrm{a}}}}$ & YN & $\mathrm{Q} / \mathrm{G}$ & $\mathrm{Q} / \mathrm{G}$ & $\mathrm{Q} / \mathrm{G}$ & $\mathrm{Q} / \mathrm{A}$ & $\mathrm{Q} / \mathrm{G}$ & $\mathrm{Q} / \mathrm{G}$ \\
\hline DD2 & 2003 & Africa & $\mathrm{Q} / \mathrm{G}$ & $\mathrm{Q} / \mathrm{H}$ & $\mathrm{Q} / \mathrm{A}$ & YN & $\mathrm{Q} / \mathrm{G}$ & $\mathrm{Q} / \mathrm{G}$ & $\mathrm{Q} / \mathrm{G}$ & $\mathrm{Q} / \mathrm{A}$ & $\mathrm{Q} / \mathrm{G}$ & $\mathrm{Q} / \mathrm{G}$ \\
\hline US-PC0082 & 2010 & USA & $\mathrm{Q} / \mathrm{G}$ & $\mathrm{Q} / \mathrm{H}$ & $\mathrm{Q} / \mathrm{A}$ & YN & $\mathrm{Q} / \mathrm{G}$ & $\mathrm{Q} / \mathrm{G}$ & $\mathrm{Q} / \mathrm{G}$ & $\mathrm{Q} / \mathrm{A}$ & $\mathrm{Q} / \mathrm{G}$ & $\mathrm{Q} / \mathrm{G}$ \\
\hline
\end{tabular}




\section{Methods}

\section{Sample collection}

Sample collection was conducted in domestic dogs at small animal hospitals in Bangkok and vicinity of Thailand During September 2016 to September 2018. 307 rectal swab samples were collected from healthy dogs $(n=55)$ and dogs with gastroenteritis symptoms $(n=252)$ including vomiting, watery diarrhea, hemorrhagic diarrhea and dehydration. The swab samples were collected from dogs of young age $(<1$ year) $(n=165)$, adult $(1-5$ years $)(n=98)$ and older ( $>5$ years) $(n=44)$. The animal demographic data including age, sex, breed, and vaccination history were also recorded. The ethics was conducted under the Chulalongkorn University's animal use and care protocol \# 1731074. The consent to participate of the owners of the animals used in this study was obtained in writing.

\section{Canine Kobuvirus (CaKoV) detection}

All 307 samples were subjected to canine Kobuvirus identification by one step RT-PCR using primers specific to 3D gene of $\mathrm{CaKoV}$ [21]. First, RNA extraction was performed using the QIAsymphony DSP Viral/Pathogen mini kit (Qiagen, Hilden, Germany) following manufacturer's instructions. To detect $\mathrm{CaKoV}$, RNA samples were screened for $3 \mathrm{D}$ gene of $\mathrm{CaKoV}$ by using one step RT-PCR assay. The primers used in this study were previously described including U1F (5'-CATGCTCCTCGGTGGTCTCA-3') and U1R (5'-GTCCGGGTCCATCACAGGGT -3') [21]. Briefly, one-step RT-PCR was conducted in a total final volume of $25 \mu \mathrm{l}$ comprising $3 \mu \mathrm{l}$ of template RNA, $15 \mu \mathrm{l}$ of 2xReaction Mix (Invitrogen, USA), $0.6 \mu \mathrm{l}$ of $10 \mu \mathrm{M}$ forward and reverse primers, $1.2 \mu \mathrm{l}$ of SuperScript III RT (Invitrogen, USA) and distilled water to final volume $25 \mu \mathrm{l}$. The condition of RT-PCR assay included cDNA synthesis step at $55^{\circ} \mathrm{C}$ for $30 \mathrm{~min}$, next to an initial denaturation step at $94^{\circ} \mathrm{C}$ for $2 \mathrm{~min}$, following 40 cycles of denaturation at $94^{\circ} \mathrm{C}$ for $30 \mathrm{~s}$, annealing at $52^{\circ} \mathrm{C}$ for $30 \mathrm{~s}$ and extension at $68^{\circ} \mathrm{C}$ for $1 \mathrm{~min}$, as well as, final extension step at $68^{\circ} \mathrm{C}$ for $5 \mathrm{~min}$. To confirm CaKoV, $4 \mu \mathrm{l}$ of PCR products were run on a $1.5 \%$ agarose gel, which mixed with Red Safe at $100 \mathrm{~V}$ for $45 \mathrm{~min}$. The expected size of $\mathrm{CaKoV}$ positive amplified products was $631 \mathrm{bp}$. Due to dogs showed clinical signs similar to other canine viral enteric diseases, all samples were also tested for Canine Parvovirus $(n=307)$, Canine Rotavirus $(n=307)$ and Canine Coronavirus $(n=30)$ [25-27].

\section{Canine Kobuvirus characterization}

In this study, four $\mathrm{CaKoV}$ positive samples (CU-53, CU101, CU-247 and CU-716) were selected for whole genome sequencing and additional eight $\mathrm{CaKoV}$ positive samples were selected for 3D and VP1 gene sequencing. The CaKoVs were selected based on epidemiological and demographic data such as age, date of isolation, breed, and vaccination history. For sequencing, nucleotide sequences of each gene of the viruses were amplified by new primer sets designed by using Primer 3 plus program [28]. List of oligonucleotide primers is provided in Additional file 1: Table S1 In brief, PCR was proceed in a final volume of $30 \mu \mathrm{l}$ containing $2 \mu \mathrm{l}$ of cDNA, $0.4 \mu \mathrm{M}$ of each forward and reverse primer, $1 \mathrm{X}$ TopTaq Master Mix, 1X Coral Load, and distilled water. The PCR condition was set as initial denaturation at $94{ }^{\circ} \mathrm{C}$ for $3 \mathrm{~min}$; 40 cycles of denaturation at $94{ }^{\circ} \mathrm{C}$ for $30 \mathrm{~s}$, annealing at $50-55^{\circ} \mathrm{C}$ for $45 \mathrm{~s}$, extension at $72^{\circ} \mathrm{C}$ for $1-$ $1.30 \mathrm{~min}$; and final extension at $72^{\circ} \mathrm{C}$ for $7 \mathrm{~min}$. PCR products were then purified and sequenced (1st Base Laboratories Sdn Bhd, Malaysia). Nucleotide sequences were edited, validated and assembled by using SeqMan software v.5.03 (DNASTAR Inc.; Wisconsin, USA).

\section{Phylogenetic and genetic analyses of canine Kobuviruses}

The phylogenetic and genetic analyses were performed by comparing nucleotide sequences of Thai CaKoVs with those of Kobuvirus available from the GenBank database. The reference nucleotide sequences of CaKoVs were retrived based on their different geographic locations, host species and date of isolation. Phylogenetic analysis of $\mathrm{CaKoV}$ was performed by using MEGA v.6.0 (Tempe, AZ, USA) [29] with neighbor-joining method with Kimura 2-parameter with 1,000 bootstrap replicates and Beast program with Bayesian Markov chain Monte Carlo (BMCMC) with 10,000,000 generations and an average standard deviation of split frequencies $<0.05$ [30]. For genetic analysis, the nucleotide sequences and deduced amino acids of $\mathrm{CaKoV}$ were aligned and compared using MegAlign software v.5.03 (DNASTAR Inc.; Wisconsin, USA). Pairwise comparison of nucleotides and amino acids of Thai $\mathrm{CaKoV}$ and those of reference $\mathrm{CaKoVs}$ were conducted. The variable and unique amino acids related to receptor binding of the viruses and host preferences of CaKoVs were monitored.

\section{Additional files}

\section{Additional file 1: Table S1. Oligonucleotide primers used for CaKoV} whole genome sequencing. (DOCX $35 \mathrm{~kb}$ )

Additional file 2: Table S2. Association of age of CaKoVs detection in this study. (DOCX $34 \mathrm{~kb}$ )

Abbreviations

CaKoV: Canine Kobuvirus; FeKoV: Feline Kobuvirus; KoV: Kobuvirus; MuKov: Murine Kobuvirus

Acknowledgements

We would like to thank the staffs of the Center of Excellence for Emerging and Re-emerging Infectious Diseases in Animals, Department of Veterinary Public Health for sample collection and data analysis. 


\section{Authors' contributions}

AA supervised and principle investigator of the project. KC, TJ, SC and RT conducted and coordinated the study, sample collection, virus identification and virus characterization. KC, NB, SB conducted data analysis and drafting the manuscript. AA drafting, revising and corresponding the manuscript. All authors read and approved the final manuscript.

\section{Funding}

This project was financial supported by the research fund under the 90th Anniversary Chulalongkorn University (Ratchadaphiseksomphot Endowment Fund) (GCUGR1125614077D). Chulalongkorn University provided financial support to the Center of Excellence for Emerging and Re-emerging Infectious Diseases in Animals for study design, sample collection, analysis and interpretation. The Thailand Research Fund supported the Royal Golden Jubilee (RGJ) Ph.D. program, for first author scholarship (RGJ-PHD/0056/2557) and TRF Senior Scholar to the corresponding author (RTA6080012)

\section{Availability of data and materials}

All data generated or analyzed during this study are included in this published article and supplement tables.

\section{Ethics approval and consent to participate}

Ethics and consent to participate in the study was conducted under the Chulalongkorn University's animal use and care protocol (IACUC) \# 1731074.

\section{Consent for publication}

The consent to participate of the owners of the animals used in this study was obtained in writing.

\section{Competing interests}

All authors in this paper declare that they have no competing interests.

\section{Author details}

${ }^{1}$ Center of Excellence for Emerging and Re-emerging Infectious Diseases in Animals, Faculty of Veterinary Science, Chulalongkorn University, Bangkok, Thailand. ${ }^{2}$ Department of Veterinary Public Health, Faculty of Veterinary Science, Chulalongkorn University, Bangkok 10330, Thailand. ${ }^{3}$ Veterinary Diagnostic Laboratory, Faculty of Veterinary Science, Chulalongkorn University, Bangkok, Thailand.

Received: 1 March 2019 Accepted: 4 July 2019

Published online: 19 July 2019

\section{References}

1. Adams MJ, Lefkowitz EJ, King AM, Harrach B, Harrison RL, Knowles NJ, Kropinski AM, Krupovic M, Kuhn JH, Mushegian AR, et al. Ratification vote on taxonomic proposals to the international committee on taxonomy of viruses (2016). Arch Virol. 2016;161(10):2921-49.

2. Oem JK, Lee MH, Lee KK, An DJ. Novel Kobuvirus species identified from black goat with diarrhea. Vet Microbiol. 2014;172(3-4):563-7.

3. Yamashita T, Ito M, Kabashima Y, Tsuzuki H, Fujiura A, Sakae K. Isolation and characterization of a new species of kobuvirus associated with cattle. J Gen Virol. 2003;84(Pt 11):3069-77.

4. Carmona-Vicente N, Buesa J, Brown PA, Merga JY, Darby AC, Stavisky J, Sadler L, Gaskell RM, Dawson S, Radford AD. Phylogeny and prevalence of kobuviruses in dogs and cats in the UK. Vet Microbiol. 2013;164(3-4):246-52

5. Kapoor A, Simmonds P, Dubovi EJ, Qaisar N, Henriquez JA, Medina J, Shields S, Lipkin WI. Characterization of a canine homolog of human Aichivirus. J Virol. 2011;85(21):11520-5.

6. Khamrin P, Maneekarn N, Kongkaew A, Kongkaew S, Okitsu S, Ushijima H. Porcine kobuvirus in piglets. Emerg Infect Dis. 2009;15(12):2075-6.

7. Li L, Victoria JG, Wang C, Jones M, Fellers GM, Kunz TH, Delwart E. Bat guano virome: predominance of dietary viruses from insects and plants plus novel mammalian viruses. J Virol. 2010;84(14):6955-65.

8. Lu G, Zhang X, Luo J, Sun Y, Xu H, Huang J, Ou J, Li S. First report and genetic characterization of feline kobuvirus in diarrhoeic cats in China. Transbound Emerg Dis. 2018;65(5):1357-63.
9. Mohamed FF, Mansour SMG, Orabi A, El-Araby IE, Ng TFF, Mor SK, Goyal SM. Detection and genetic characterization of bovine kobuvirus from calves in Egypt. Arch Virol. 2018;163(6):1439-47.

10. Phan TG, Kapusinszky B, Wang C, Rose RK, Lipton HL, Delwart EL. The fecal viral flora of wild rodents. PLoS Pathog. 2011;7(9):e1002218.

11. Li L, Pesavento PA, Shan T, Leutenegger CM, Wang C, Delwart E. Viruses in diarrhoeic dogs include novel kobuviruses and sapoviruses. J Gen Virol. 2011;92(Pt 11):2534-41.

12. Di Martino B, Di Felice E, Ceci C, Di Profio F, Marsilio F. Canine kobuviruses in diarrhoeic dogs in Italy. Vet Microbiol. 2013;166(1-2):246-9.

13. Kong N, Zuo Y, Wang Z, Yu H, Zhou EM, Shan T, Tong G. Molecular characterization of new described kobuvirus in dogs with diarrhea in China. Springerplus. 2016;5(1):2047.

14. Oem JK, Choi JW, Lee MH, Lee KK, Choi KS. Canine kobuvirus infections in Korean dogs. Arch Virol. 2014;159(10):2751-5.

15. Soma T, Matsubayashi M, Sasai K. Detection of kobuvirus RNA in Japanese domestic dogs. J Vet Med Sci. 2016;78(11):1731-5.

16. Olarte-Castillo XA, Heeger F, Mazzoni CJ, Greenwood AD, Fyumagwa R, Moehlman PD, Hofer H, East ML. Molecular characterization of canine kobuvirus in wild carnivores and the domestic dog in Africa. Virology. 2015;477:89-97.

17. Bodewes R, Ruiz-Gonzalez A, Schapendonk CM, van den Brand JM, Osterhaus AD, Smits SL. Viral metagenomic analysis of feces of wild small carnivores. Virol J. 2014;11:89.

18. Di Martino B, Di Profio F, Melegari I, Robetto S, Di Felice E, Orusa R, Marsilio F. Molecular evidence of kobuviruses in free-ranging red foxes (Vulpes vulpes). Arch Virol. 2014;159(7):1803-6.

19. Melegari I, Sarchese V, Di Profio F, Robetto S, Carella E, Bermudez Sanchez S, Orusa R, Martella V, Marsilio F, Di Martino B. First molecular identification of kobuviruses in wolves (Canis lupus) in Italy. Arch Virol. 2018;163(2):509-13.

20. Ribeiro J, Headley SA, Diniz JA, Pereira AH, Lorenzetti E, Alfieri AA, Alfieri AF. Extra-intestinal detection of canine kobuvirus in a puppy from southern Brazil. Arch Virol. 2017;162(3):867-72.

21. Choi JW, Lee MH, Lee KK, Oem JK. Genetic characteristics of the complete feline kobuvirus genome. Virus Genes. 2015;50(1):52-7.

22. Li M, Yan N, Wang M, Zhang B, Yue H, Tang C. Prevalence and genomic characteristics of canine kobuvirus in Southwest China. Arch Virol. 2018;163(2):459-66.

23. Li CQ, Wei S, Guo DH, Wang ZH, Geng YF, Wang EY, Zhao XW, Su MJ, Wang XY, Sun DB. Prevalence and phylogenetic analysis of canine kobuviruses in diarrhoetic dogs in Northeast China. J Vet Med Sci. 2016;78(1):7-11.

24. Chen L, Zhu L, Zhou YC, Xu ZW, Guo WZ, Yang WY. Molecular and phylogenetic analysis of the porcine kobuvirus VP1 region using infected pigs from Sichuan Province. Virol J. 2013;10:281.

25. Pratelli A, Tempesta M, Greco G, Martella V, Buonavoglia C. Development of a nested PCR assay for the detection of canine coronavirus. J Virol Methods. 1999;80(1):11-5.

26. Buonavoglia C, Martella V, Pratelli A, Tempesta M, Cavalli A, Buonavoglia D, Bozzo G, Elia G, Decaro N, Carmichael L. Evidence for evolution of canine parvovirus type 2 in Italy. J Gen Virol. 2001;82(Pt 12):3021-5.

27. Ortega AF, Martinez-Castaneda JS, Bautista-Gomez LG, Munoz RF, Hernandez IQ. Identification of co-infection by rotavirus and parvovirus in dogs with gastroenteritis in Mexico. Braz J Microbiol. 2017;48(4):769-73.

28. Koressaar T, Remm M. Enhancements and modifications of primer design program Primer3. Bioinformatics. 2007;23(10):1289-91.

29. Tamura K, Stecher G, Peterson D, Filipski A, Kumar S. MEGA6: molecular evolutionary genetics analysis version 6.0. Mol Biol Evol. 2013;30(12):2725-9.

30. Drummond AJ, Suchard MA, Xie D, Rambaut A. Bayesian phylogenetics with BEAUti and the BEAST 1.7. Mol Biol Evol. 2012;29(8):1969-73.

\section{Publisher's Note}

Springer Nature remains neutral with regard to jurisdictional claims in published maps and institutional affiliations. 